\title{
Tunable Transmission Filters Based on Corrugated Sidewall Bragg Gratings in Polymer Waveguides
}

\author{
Lin Zhu, Yanyi Huang, William M.J. Green, Amnon Yariv \\ Department of Electrical Engineering, Department of Applied Physics, California Institute of Technology, Pasadena, California 91125 \\ linzacaltechedu
}

\begin{abstract}
We fabricate different bandpass filters based on polymeric waveguide gratings by introducing phase shift defects and integrating with a $3 \mathrm{~dB}$ MMI coupler. We also use thermo-optic effects to dynamically tune the designed filter.

(C)2005 Optical Society of America

OCIS codes: (350.2770) Gratings; (230.7390) Waveguides, Planar
\end{abstract}

\section{Introduction}

In recent years, low cost bandpass filters have become key components for wavelength division multiplexing (WDM) metro networks. Optical filters based on Bragg gratings have been widely investigated in photosensitive fibers[1-2], semiconductor waveguides[3] and polymer waveguides[4]. However, most designs are combined with a optical circulator since Bragg gratings are intrinsically reflection devices. The usage of a circulator will cause integration problems and cost considerations. One alternative solution is to introduce phase shift defects in Bragg gratings, and another is to integrate Bragg gratings on the arm of an optical coupler. Planar polymeric waveguide devices, such as directional couplers, Mach-Zehnder interferometers, ring resonators, and Bragg grating reflectors are becoming increasingly attractive for optical integrated circuits owing to the low cost and mechanical flexibility of polymer materials. Thus, it is very promising to incorporate Bragg gratings of simple bandpass design within polymer waveguides for making low cost transmission filters.

Currently, polymeric Bragg grating filters are primarily fabricated by exposing the polymer with UV or visible light through a pre-designed phase mask. During the past two decades, electron beam lithography has been widely applied in fabricating complex micro/nano structures. In this paper, we apply direct electron beam writing to make corrugated sidewall Bragg gratings in polymer waveguides and demonstrate transmission filters with different bandwidth by introducing phase shift defects and integrating with a multi-mode interferometer (MMI) coupler.

2. Phase-Shifted Grating Design, Fabrication and Measurement
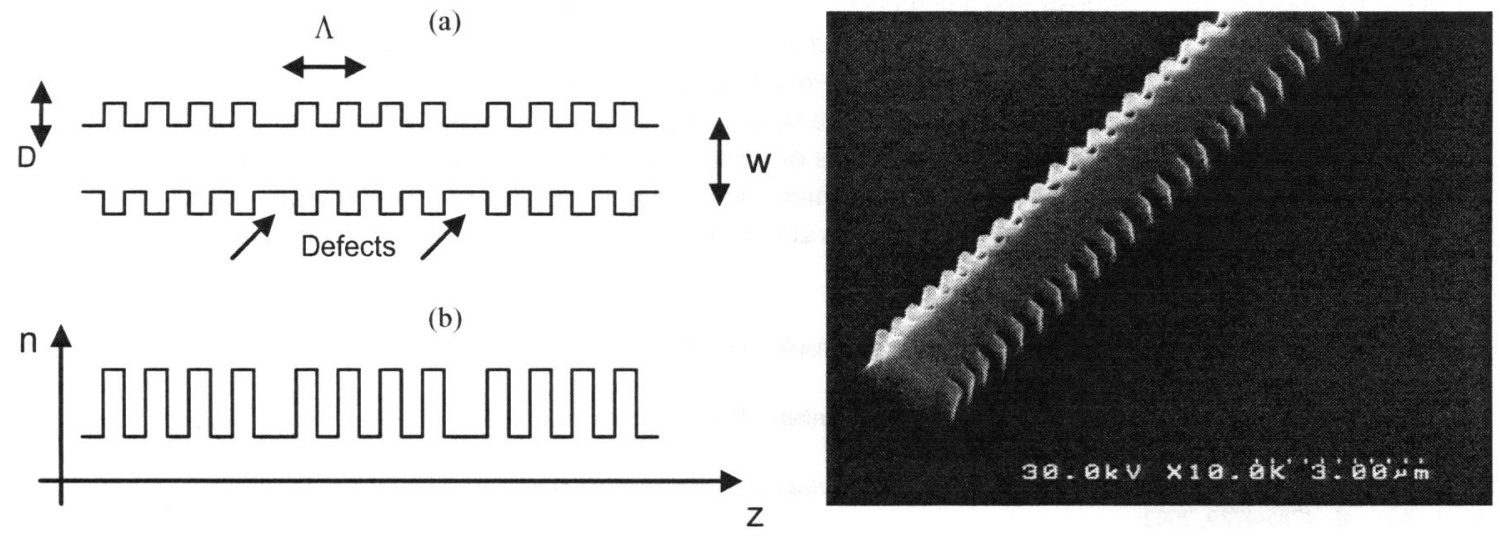

Fig.1. Schematic diagrams of (a) polymeric waveguide gratings with two phase shift defects, and (b) the corresponding effective refractive index profile along the propagation direction. (c) SEM image of a polymeric grating with a waveguide width $\mathrm{W}$ of $1.5 \mu m$, grating period $\Lambda$ of $500 \mathrm{~nm}$, and grating depth $\mathrm{D}$ of $600 \mathrm{~nm}$ 


\section{CMP6}

Figure 1 shows the schematic diagram of a corrugated sidewall Bragg waveguide grating with the corresponding effective refractive index profile and a scanning electron microscope (SEM) image of a polymeric grating with a waveguide width $\mathrm{W}$ of $1.5 \mu \mathrm{m}$, grating period $\Lambda$ of $500 \mathrm{~nm}$, and grating depth $\mathrm{D}$ of $600 \mathrm{~nm}$. In Fig. 1(a), the uniform gratings are formed by introducing periodic vertical grooves along the waveguide sidewall. By appropriately changing the groove depth $\mathrm{D}$, it is possible to achieve a significant relative refractive index modulation $\Delta n / n$ of $\sim 0.001-0.1$. The $\pi$ phase shift can be created by introducing a defect in the periodic structure, corresponding to a quarter wavelength discontinuity. To fabricate the device in Fig. 1(c), a $1.6 \mu m$ thick optical core layer of the negative electron beam resist SU-8 is first spun onto a silicon wafer with $5 \mu \mathrm{m}$ of thermally grown silicon oxide, which serves as the lower cladding. Then, waveguides with corrugated sidewall gratings are directly patterned by electron beam exposure using a SEM. The crosslinked SU-8 exhibits a relatively high refractive index $(\mathrm{n}=1.565$ at $1550 \mathrm{~nm})$.

Figure 2 shows the spectral response of a multi-channel passband filter based on a polymer waveguide grating with two intentionally introduced $\pi$ phase shifts. The device structure is very similar to that shown in Fig. 1(a). The defects are separated by a $90 \mu m$ segment of uniform grating, and the length of the device is $720 \mu m$. The fabricated gratings have the same parameters as in Figure 1. In Fig. 2(a), the bandwidth of the stopband and the passband are $5.2 \mathrm{~nm}$ and $0.8 \mathrm{~nm}$, respectively. The spectrum in Fig. 2(b) is obtained after applying a $\sim 10 \mu m$ thick layer of index matching fluid $(\mathrm{n}=1.44$ at $1550 \mathrm{~nm})$ over the top of the original device. In Fig 2 (b), comparing the experimental data to the fitting results shows that the measured transmission in the passband channels is lower than expected, and that the stopband minimum is higher. These discrepancies are attributed to scattering loss/fabrication errors and photodetector dark current/undesired laser scattering, respectively.
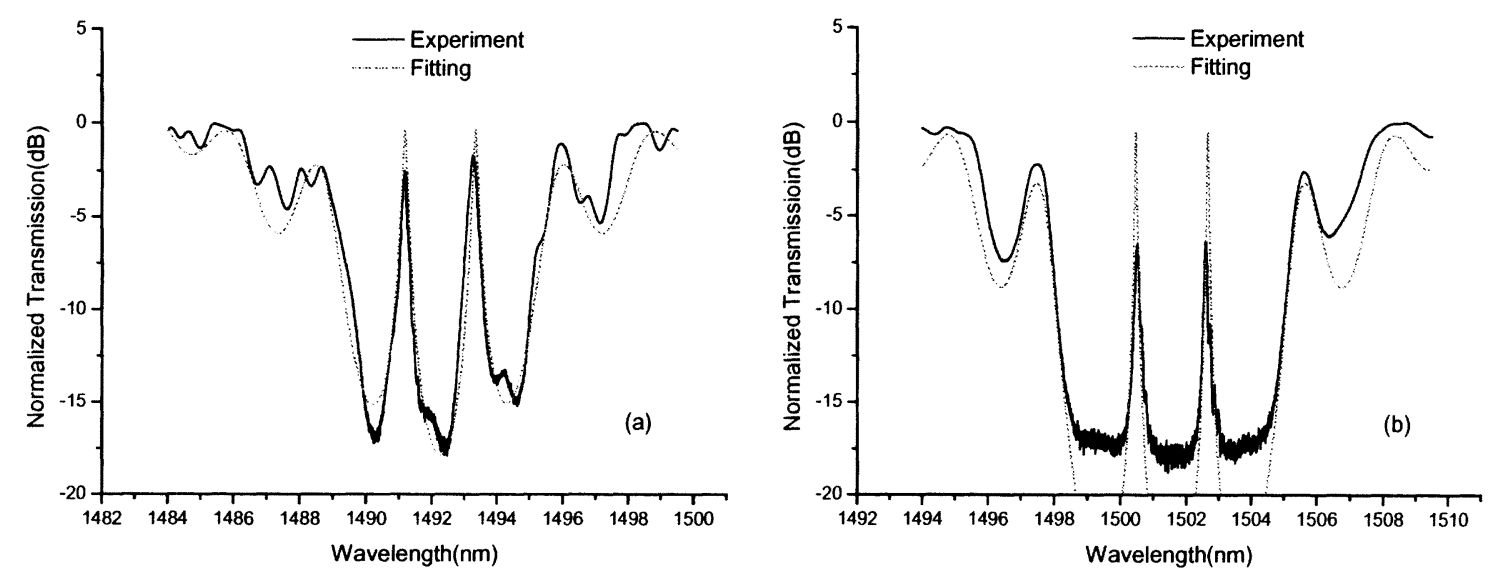

Fig.2. Transmission spectrum of a passband filter with phase shift defects for (a) without the index matching fluid, $n_{e f f}=1.4923$ and $\Delta n=0.0050$ from fitting results (b) with the index matching fluid as upper cladding, $n_{\text {eff }}=1.5016$ and $\Delta n=0.0070$ from fitting results.

The index matching fluid serves two main functions. First, it eliminates the reflection from the two cleaved end facets of the device, and the associated Fabry-Perot resonances observed in Fig. 2(a). Second, it acts as an upper cladding material for the waveguide gratings. In Fig. 2(b), the central wavelength of the device is longer than in Fig. 2(a). This is because the additional upper cladding material causes the modal effective index to increase. We also find that the stopband is broader and the passband is sharper. The bandwidth of stopband and passband are $6.8 \mathrm{~nm}$ and $0.4 \mathrm{~nm}$, respectively, which is due to a larger effective refractive index modulation experienced by the cladded device in Fig. 2(b). This larger $\Delta n$ is also caused by the upper cladding fluid, since the optical mode is less confined after applying the upper cladding. Thus, the groove features would have a more significant impact on the mode in the waveguide. In fact, it provides us a simple but effective method to post tune the filter spectral response through the use of upper cladding materials with different refractive indexes. 


\section{CMP6}

\section{Integration of waveguide gratings with a MMI coupler and thermal tuning}


Fig.3. (a) Transmission spectrum of a passband filter based on the integration of waveguide gratings with a $3 \mathrm{~dB}$ MMI coupler at room temperature, (b) dependence of the central wavelength and bandwidth of the device on temperature

Fig. 3(a) shows the schematic diagram of the fabricated bandpass filter based on the integration of waveguide gratings with a MMI coupler and the corresponding transmission spectrum. The waveguide gratings have a waveguide width $\mathrm{W}$ of $1.5 \mu \mathrm{m}$, grating period $\Lambda$ of $520 \mathrm{~nm}$, and grating depth $\mathrm{D}$ of $500 \mathrm{~nm}$. The MMI coupler is carefully designed to obtain a 50/50 coupling ratio between two up arms in order to minimize transmission loss. Comparing to Figure 2, the transmission spectrum of the filter has a longer central wavelength and a smaller bandwidth, since we use a longer grating period and a smaller grating depth for the device in Figure 3.

The large thermo-optic coefficient $\mathrm{dn} / \mathrm{dT}$ (refractive index change with temperature) of polymer materials implies that we can dynamically tune the grating filter by changing the temperature. Fig. 3(b) shows the dependence of the central wavelength and bandwidth of the fabricated device. From $24^{\circ} \mathrm{C}$ to $86^{\circ} \mathrm{C}$, we get a tuning range of $6.2 \mathrm{~nm}$ for the filter central wavelength, which corresponds to an average thermo-optic coefficient of $-9.6 \times 10^{-5} /{ }^{\circ} \mathrm{C}$. When the temperature is beyond $60^{\circ} \mathrm{C}$, the changing rate of central wavelength decreases a little bit due to the saturation. In Fig. 3(b), we also notice that the filter bandwidth varies slightly in a range between $2.9 \mathrm{~nm}$ to $3.2 \mathrm{~nm}$ when the temperature changes. This is because that the change of waveguide core index will also lead to the variation of index modulation of the grating.

\section{Conclusion}

We use electron beam direct writing to fabricate a passband filter in a polymer waveguide with phase-shifted corrugated sidewall Bragg gratings and integrate an uniform waveguide grating on the arm of a $3 \mathrm{~dB}$ MMI coupler to make another type of transmission filter. We also use thermo-optic effects to tune the integrated bandpass filter and obtain a tuning range of $6.2 \mathrm{~nm}$ with a temperature change of $62^{\circ} \mathrm{C}$.

\section{Reference:}

[1]J.Canning et al, "phase-shifted periodic distributed structure in optical fibers by UV post processing," Electron. Lett. 30, 1344-1345 (1994).

[2]K.Sugden et al, "Fabrication and characterization of bandpass filters based on concatenated chirped fiber gratings," J. Lightwave Technol. 15, 1433-1437 (1997)

[3]T.Segawa, et al, “Apodised sampled grating using InGaAsP/InP deep-ridge waveguide with vertical-groove surface grating," Electron. Lett. 40, 804-805 (2004).

[4]M.C.Oh et al, "Polymeric wavelength filters with polymer gratings," Appl. Phys. Lett. 72, 1559-1561(1998).

[5]A.Yariv, P.Yeh, Optical waves in crystals, Wiley Interscience, 2003. 